\title{
Review \\ Antigen receptor signaling in the rheumatic diseases
} Julie Zikherman and Arthur Weiss

Division of Rheumatology, Rosalind Russell Medical Research Center for Arthritis, Department of Medicine, Howard Hughes Medical Institute, University of California, San Francisco, 513 Parnassus Avenue San Francisco, CA 94143, USA

Corresponding author: Arthur Weiss, aweiss@medicine.ucsf.edu

Published: 30 January 2009

(c) 2009 BioMed Central Ltd
This article is online at http://arthritis-research.com/content/11/1/202

Arthritis Research \& Therapy 2009, 11:202 (doi:10.1186/ar2528)

some of these advances and propose some general principles that tie these observations together.

Antigen receptor signaling in lymphocytes has been clearly implicated in the pathogenesis of the rheumatic diseases. Here, we review evidence from mouse models in which B-cell and T-cell signaling machinery is perturbed as well as data from functional studies of primary human lymphocytes and recent advances in human genetics. B-cell receptor hyper-responsiveness is identified as a nearly universal characteristic of systemic lupus erythematosus in mice and humans. Impaired and enhanced T-cell receptor signaling are both associated with distinct inflammatory diseases in mice. Mechanisms by which these pathways contribute to disease in mouse models and patients are under active investigation.

\section{Introduction}

The classic concept of autoimmune disease rests upon the notion that the adaptive immune system generates inappropriate antigen-specific responses to self epitopes which in turn drive disease. Indeed, the presence of autoantibodies is one of the most characteristic features of the rheumatic diseases. Since the canonical definition of the adaptive immune response relates to the ability of somatic recombination to produce an enormous range of antigen receptors on lymphocytes, it follows that antigen receptor signal transduction ought to play a role in autoimmune diseases. The Tcell antigen receptor (TCR)-beta chain was cloned in 1983, and the subsequent decade saw the discovery of the signal transduction pathway downstream of the TCR [1]. Parallel discoveries for B-cell antigen receptor (BCR) signaling followed. Not only antigen receptors themselves but the complex machinery that elaborates the cellular response to antigen have been implicated in the rheumatic diseases. The past decade has seen evidence confirm this view from a range of sources, including engineered and spontaneous mouse models, primary lymphocytes from patients, as well as human genetics. Here, we provide a selective overview of

\section{Overview of antigen receptor signal transduction}

TCR signal transduction is initiated following interaction of the TCR $\alpha \beta$ chains with peptide antigen bound to major histocompatibility complex (MHC) class I or II molecules. The signal is transmitted to a complex network of kinases, phosphatases, and adaptors (Figure 1). The TCR $\alpha \beta$ chains lack any ability to transmit signals on their own and depend on $\operatorname{CD} 3(\varepsilon, \delta$, and $\gamma)$ and $\zeta$ chains that contain varying numbers of immunoreceptor tyrosine-based activating motifs (ITAMs). The dual tyrosines of ITAMs are phosphorylated by the Src family kinases (SFKs), which, in T cells, are Lck and Fyn. Dually phosphorylated ITAMs, in turn, form docking sites for the tandem SH2 domains of Syk family kinases, ZAP-70 and Syk. The Syk kinases are activated upon binding to phospho-ITAMs and phosphorylation by the SFKs. Once activated, the Syk kinases phosphorylate the critical adaptors Slp-76 and Lat, which together form the scaffolds for assembly of further signaling molecules. Among these is the enzyme phospholipase C $\gamma 1$ (PLC $\gamma 1$ ), which is responsible for transmission of signals to phosphorylate mitogenactivated protein kinases (MAPKs) and increase cytoplasmic free calcium. Functional consequences of antigen receptor signaling are varied and context-dependent, including cell activation, proliferation, differentiation, and death [2,3].

In addition to antigen binding, there are many levels of regulation in this signaling pathway. The SFKs themselves are tightly regulated by phosphorylation of their inhibitory Cterminal tyrosine residue. Reciprocal regulation of this phosphotyrosine by the receptor-like tyrosine phosphatase CD45 and the cytoplasmic kinase Csk can set thresholds for

$\mathrm{ANA}=$ anti-nuclear antibody; $\mathrm{BCR}=\mathrm{B}$-cell antigen receptor; $\mathrm{CR} 2=$ complement receptor-2; $\mathrm{dKO}=$ double knockout; dsDNA $=$ double-stranded DNA; IL = interleukin; ITAM = immunoreceptor tyrosine-based activating motif; ITIM = immune tyrosine inhibitory motif; Me ${ }^{v}=$ moth-eaten viable; $\mathrm{MHC}=$ major histocompatibility complex; PLC $\gamma 1=$ phospholipase $\mathrm{C} \gamma 1 ; \mathrm{RA}=$ rheumatoid arthritis; SFK $=$ Src family kinase; SLE = systemic lupus erythematosus; $\mathrm{TCR}=\mathrm{T}$-cell antigen receptor; $\mathrm{Tg}=$ transgene; $\mathrm{Treg}=$ regulatory $\mathrm{T}$ cell. 
Figure 1

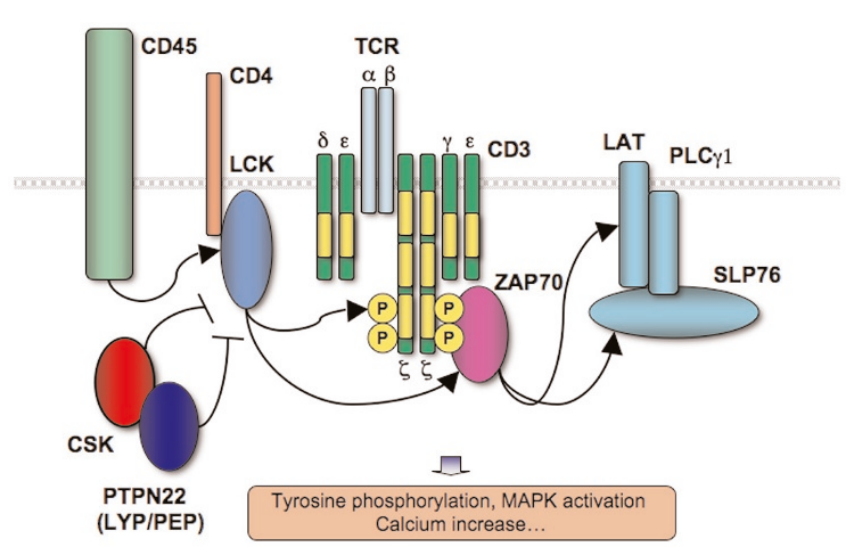

Schematic representation of T-cell receptor signal transduction. CD4-associated Lck is reciprocally regulated by CD45 and Csk/PTPN22 and in turn phosphorylates CD3 chain immunoreceptor tyrosine-based activating motifs (ITAMs) and ZAP-70. ZAP-70 phosphorylates additional downstream effectors, including the adaptors SIp-76 and Lat. Yellow bands represent CD3 chain ITAM domains. Phosphotyrosines are not depicted on all CD3 chain ITAMs. MAPK, mitogen-activated protein kinase; PLC $\gamma 1$, phospholipase C $\gamma 1$; TCR, T-cell antigen receptor.

antigen receptor signal transduction. Added complexity is presented by tight regulation of the activating tyrosine of the SFKs. Negative regulators of TCR signaling, such as the phosphatases Pep and SHP-1, can dephosphorylate this critical residue $[4,5]$.

The BCR immunoglobulin chains are responsible for antigen recognition (Figure 2). BCR signal transduction resembles TCR signaling in many ways, relying upon the ITAMs of the associated $\lg \alpha$ and $\lg \beta$ chains, the B-cell-expressed SFKs Lyn, Fyn, and Blk, and the Syk kinase as well as homologous adaptors (Blnk/Slp-65 instead of Slp-76). CD45 and Csk also regulate SFKs in B cells as they do in T cells [6].

Multiple pathways feed into this network at several proximal signaling nodes, including positive and negative regulators of antigen receptor signaling. In $\mathrm{T}$ cells, for instance, the coreceptors CD4 and CD8 play a positive regulatory role not only by facilitating $\mathrm{MHC}$ recognition, but also by bringing the SFK Lck into the proximity of the TCR [2]. The complex of CD19/CD81/CD21 (CR2, complement receptor-2) that interacts with the Lyn SFK plays a similar coreceptor role on $B$ cells. These coreceptors are counterbalanced by the action of receptors with negative regulatory function. Cell surface molecules that exert negative regulation often contain a cytoplasmic motif, called an ITIM (immune tyrosine inhibitory motif), which upon phosphorylation by SFKs recruits negative regulators of signaling, such as the protein tyrosine phosphatases SHP-1 and SHP-2 and the lipid phosphatase SHIP. Such ITIM-containing receptors are best characterized
Figure 2

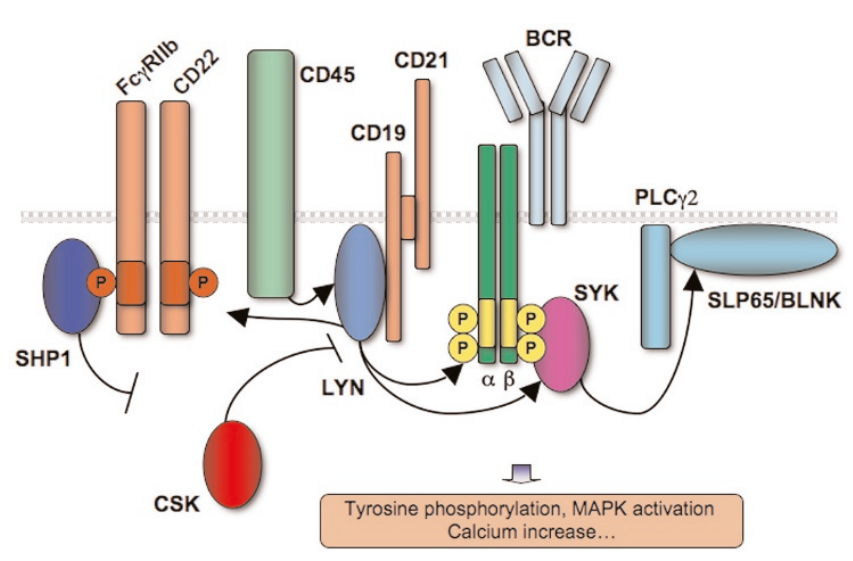

Schematic representation of B-cell receptor signal transduction. Lyn is reciprocally regulated by $\mathrm{CD} 45$ and Csk and in turn phosphorylates B-cell antigen receptor (BCR) immunoreceptor tyrosine-based activating motifs (ITAMs) as well as immune tyrosine inhibitory motif (ITIM)-containing immunoreceptors. Positive and negative signals are in turn transmitted by Syk and SHP-1, respectively. Yellow bands on $\lg \alpha$ and $\beta$ chains represent ITAM domains. Orange bands on CD22 and Fc $\gamma R$ Rllb represent ITIM domains. MAPK, mitogen-activated protein kinase; PLC $\gamma 2$, phospholipase $\mathrm{C} \gamma 2$.

in B cells and natural killer cells. Inhibitory phosphatases, once localized to the plasma membrane by phosphorylated ITIMs, are placed in proximity to ITAM-containing receptors and, in turn, negatively regulate their function. CD22 and FcyRIlb are examples of B-cell-specific ITIM-containing surface receptors that are critical modulators of BCR signaling $[7,8]$. Inhibitory cell surface molecules such as PD-1 and CTLA-4 are expressed on $\mathrm{T}$ cells and analogously modulate TCR signal transduction, although only PD-1 contains a canonical ITIM [9]. Despite abundant similarities, wiring differs critically between $T$ and $B$ cells and among distinct stages of lymphocyte development. Most notably, the Lyn SFK in B cells is felt to play a non-redundant negative regulatory role downstream of numerous ITIM-containing receptors [10]. A homologous 'negative' role for Lck or Fyn in $\mathrm{T}$ cells has yet to be clearly demonstrated.

\section{Antigen receptor signaling in lymphocyte development}

Studies in mice have revealed that antigen receptor signaling is critical not only in the response of mature lymphocytes to foreign antigen but in the progression of lymphocytes through a series of developmental stages in which both liganddependent and ligand-independent signals are required to proceed. Perhaps most significantly, antigen receptor signaling is necessary for 'testing' and refining the antigen receptor repertoire during development. Candidate TCRs are tested in the thymus for 'just right' signal strength by positive and negative selection. Perturbations in TCR signal transduction influence this process [11]. Analogous processes have been 
identified in B cells in the bone marrow and the periphery [12]. Thymic lineage decisions have been shown to critically depend upon antigen receptor signal strength, including Foxp $3^{+}$regulatory $T$ cell (Treg) fate [13]. Antigen receptor signaling in the periphery is also critical in the maintenance of immune homeostasis and tolerance to self. These antigen receptor-dependent events are likely relevant for the interpretation of disease pathogenesis in signaling mutants.

\section{Mouse models}

An extensive mouse model literature can teach us about the signaling requirements for tolerance and autoimmunity. Evidence for the role of antigen receptor signaling in autoimmunity and insight into disease pathogenesis comes from both forward and reverse genetic approaches, involving both engineered and spontaneous mutations. Our approach here is to group mutations with similar functional consequences (hypo- or hyper-responsiveness) in T cells or B cells and to explore links to disease.

\section{B-cell antigen receptor signaling mutants and murine lupus}

Several single-gene mutants develop a lupus-like disease characterized by the production of anti-nuclear antibodies (ANAs) in the context of hyper-responsive BCR signaling. Examples include FcyRllb-/-, Lyn-/-, Lynup/up, CD45 E613R, $\mathrm{CD}^{2}{ }^{-/-}$, CD19 transgenic (Tg), and SHP-1 (Mev) mice (see [14] for detailed review). These mutations, in turn, can be grouped into functional pathways. CD22, FcyRllb, and SHP-1 are exclusively negative regulators of $\mathrm{BCR}$ signaling [6]. The moth-eaten viable allele of SHP-1 $\left(\mathrm{Me}^{v}\right)$ is a spontaneously arising hypomorph with reduced phosphatase activity [14]. The SFK Lyn plays a more complex role in BCR signal transduction [10]. A confusing observation has been that two opposing alleles of Lyn (Lyn-/- and Lynup/up) both produce Bcell hyper-responsiveness and ANAs. This strongly suggests that Lyn has both positive and negative regulatory roles. Lyn is critical for BCR signal transduction as well as function of inhibitory coreceptors such as FcyRIlb and CD22. Lyn exerts its negative regulatory role by phosphorylating ITIMs that in turn recruit SHP-1 and SHIP. Lyn is thought to subserve this function in a non-redundant fashion despite expression of two other SFKs in B cells, Fyn and Blk. CD19 is a B-cell-specific cell surface protein that forms the signaling component of the CR2 complement receptor (CD21) in conjunction with CD81 [6]. CD19 contains multiple tyrosines and positively regulates $\mathrm{BCR}$ signal transduction. Its overexpression in mice leads to the production of autoantibodies [15]. E613R is a dysregulated allele of CD45, which in turn influences SFK activity. Mice harboring this mutation develop a lymphoproliferative syndrome and a lupus-like disease on permissive genetic backgrounds [16]. The disease is driven by $B$ cells that are extremely hyper-responsive to BCR signaling [17].

The characteristics of the disease(s) in these animals are interesting. All produce autoantibodies but their specificities vary. CD22 $2^{-/-}$mice produce anti-cardiolipin antibodies and anti-myeloperoxidase antibodies, whereas CD19 Tg mice produce single-stranded DNA antibodies as well as rheumatoid factor $[15,18]$. This observation suggests that there may be a common general mechanism for autoantibody production in various autoimmune diseases. It has recently been demonstrated that the innate pattern recognition receptors TLR7 and TLR9 are critical (and sufficient in a B-cellintrinsic manner) to generate antibodies to DNA/nuclear components broadly and to direct specificities as well [19]. It is likely that BCR signal transduction cooperates with this pathway. Whether other factors cooperate and which ones do so are still unknown. This exciting discovery complicates conventional distinctions between innate and adaptive responses and undercuts assumptions about clonal escape from tolerizing mechanisms.

A general feature of this collection of mouse models is that genetic background effects are very significant. FcyRllb develops lupus-like disease on the B6 background but not on the Balb/c background [20]. CD45 E613R mice, in contrast, remain healthy with no ANAs on the B6 background, whereas on the Balb/c background $100 \%$ of the animals develop ANAs (M Hermiston, V Lam, R Mills, N Oksenberg, N Cresalia, A Tam, M Anderson and A Weiss, manuscript in preparation). Furthermore, a number of these models can produce disease on a 'non-autoimmune' background in the setting of cooperating mutations [20].

When and how is tolerance broken in these mice? The answer to this question is exceedingly complex because many of these models influence cell lineages other than $B$ cells. Indeed, genetic deletion of lymphocytes in $\mathrm{Me}^{v}$ mice does not fully rescue disease, suggesting that myeloid cellintrinsic defects can drive the motheaten phenotype [21].

To understand how and where enhanced BCR signal transduction produces autoantibodies, we will focus our attention on those mice in which signaling is perturbed only in B cells. We are left with the CD22-l-, FcyRllb-/-, and CD19 Tg models. FcyRllb ${ }^{-/}$is the most extensively studied of these, and crosses to BCR transgenes have revealed that the tolerance break is peripheral and 'late' [22]. Similarly, CD22 is expressed and influences signaling in a relatively narrow pattern upon mature conventional B cells [14]. Lyn ${ }^{-1-}$ (Andrew Gross, personal communication) and CD45 E613R (J Zikherman, M Hermiston, D Steiner, K Hasegawa, A Chan, A Weiss, manuscript in preparation) $B$ cells also exhibit hyperresponsiveness to $\mathrm{BCR}$ stimulation predominantly at the follicular mature peripheral B cell stage of development. Taken together, these data suggest that peripheral BCR hyper-responsiveness cooperates with other events (such as TLR signaling) to break tolerance by accelerating differentiation into plasma cells or progression into germinal centers. It may be that sufficient 'escape' of anti-nuclear B cells to the periphery occurs physiologically [23]. A 'central' 
tolerance break may not be necessary in these mouse models of lupus.

In other mouse models, both a central and peripheral tolerance break may be required. The NZB/W mouse is a spontaneous polygenic model of lupus which has been studied extensively over the last 20 years. Genetically separable cellular phenotypes resemble those seen in engineered models of lupus. For example, BCR hyper-responsiveness maps to the lupus-prone NZM2410-derived Sle2 genetic locus but cannot independently produce disease [24]. The Sle1 region is associated with the appearance of ANAs [25]. Sle1 recently was mapped to Ly108, a member of the SLAM family of receptors that signal through a non-ITAM/ITIM pathway that relies upon the adaptor SAP and the SFK Fyn [26]. Ly108 is highly expressed in immature $B$ cells and can modulate BCR signal strength. The NZB/W-derived allele of Ly108 produces weaker BCR signaling than the B6 allele in immature B cells. This allele may act early during negative selection of $B$ cells, permitting polyreactive anti-doublestranded DNA (anti-dsDNA) B cells to escape to the periphery.

Thus, opposing signaling phenotypes may be required to breach 'central' and 'peripheral' tolerance mechanisms. The two may even coexist in the same animal as genetically separable phenotypes as demonstrated by the NZB/W lupus model. Whether analogous functional phenotypes characterize human systemic lupus erythematosus (SLE) will be interesting to determine.

\section{Proximal T-cell antigen receptor signal transduction and autoimmune disease}

There are many examples of signaling mutants in which proximal TCR signaling machinery is impaired, and a number of these mutants develop disease. The Skg mouse model of rheumatoid arthritis (RA) is due to a spontaneous mutation that arose in an inbred colony of Balb/c mice [27]. These animals develop a destructive polyarthritis associated with rheumatoid factor and anti-cyclic citrullinated peptide antibody production. The mutation was identified as a single amino acid substitution in ZAP-70 (W613C). This mutation impairs ZAP-70 association with TCR $\zeta$-chain ITAMs and results in markedly reduced TCR signal transduction. The mice exhibit impaired positive and negative selection in the thymus as well as a hypo-proliferative phenotype in the periphery. Consistent with a ZAP-70 mutation, the disease is T-cell-mediated; CD4 T cells, but not serum, can transfer the disease, even into $\mathrm{RAG}^{-/-}$hosts lacking endogenous $\mathrm{T}$ and $\mathrm{B}$ cells [28].

The pathogenesis of disease in these animals remains unclear [28]. Impaired negative selection has been observed and may permit autoreactive $\mathrm{T}$ cells to escape to the periphery. However, a bona fide autoantigen has not yet been identified. Other potential etiologies of disease include abnormalities in Tregs, which are reduced in number and impaired in function. Whether Tregs play a critical role in the pathogenesis of Skg arthritis is still uncertain. Cytokine milieu appears to be perturbed in these animals, and dysregulated T-cell differentiation and cytokine production may play an important role. Indeed, crosses to cytokine knockouts have shown that IL-6 and IL-17, but not interferon-gamma, are required to mediate disease. Interestingly, the disease disappeared in a clean specific-pathogen-free facility but could be induced by innate immune stimulation of pattern recognition receptors by dectin, a fungal cell wall component [29]. Thus, the dysregulated immune system in these animals has to be tipped over the edge, so to speak.

An informative allelic series of ZAP-70 hypomorphic mutants was described recently and provided an opportunity to study graded TCR signaling and its role in autoimmunity [30]. The ZAP-70 allelic series revealed a threshold effect in which partial, but neither mild nor severe, T-cell immunodeficiency was sufficient to break tolerance. Partially impaired TCR signal transduction was associated with the appearance of ANAs as well as hyper-lgE and $\lg \mathrm{G} 1$ antibody production. The latter suggests an unusual Th2 polarization, which we will mention again below in the context of other mutants.

This phenotype did not resemble the ZAP-70 hypomorphic Skg allele. The ZAP-70 allelic series was generated on the B6 genetic background, whereas the Skg ZAP-70 allele leads to arthritis only on the Balb/c background in the context of an innate immune stimulus. A common target molecule with quantitatively or qualitatively impaired TCR signaling may provoke different diseases in different genetic and environmental contexts, as seen with B-cell perturbations. The mouse models discussed above include quantitative and perhaps qualitative impairments in a single critical molecule, ZAP-70, involved in TCR signal transduction. What of perturbations in distinct signaling pathways downstream of the TCR?

The Lat Y136F mutation eliminates binding of PLC $\gamma 1$ to a critical phospho-tyrosine of the Lat adaptor [31,32]. T cells from Lat Y136F mice exhibit profoundly impaired calcium flux with relatively preserved Erk phosphorylation. Thymic development is perturbed with a partial block at beta selection as well as positive selection. At 2 to 3 weeks of age, the mice develop a lymphoproliferative disorder characterized by CD4 T-cell expansion and overproduction of Th2 cytokines. The mice exhibit associated polyclonal B-cell activation and elevation of $\lg$ E and $\lg$ G1. An inflammatory disease develops with multiorgan infiltrates and production of ANAs as well as dsDNA antibodies. The phenotype is much more severe than that associated with the ZAP-70 allelic series, but the unusual development of Th2 cytokine overproduction (and hyper-IgE levels) is reminiscent.

Taken together, the pathogenesis of autoimmunity in these models is not obvious [33]. Clearly, impaired signal transduction perturbs thymic selection and influences the T-cell 
repertoire. However, as in the Skg mouse, initiating autoantigens have not been identified and how an autoantigen could stimulate severely signaling-impaired peripheral $\mathrm{T}$ cells to produce disease is unclear. Lineage commitment in the thymus is also perturbed; Treg development and function are abnormal. Indeed, a number of mouse models with impaired TCR signaling and autoimmunity are rescued by transfer of wild-type Tregs [33]. However, the ability of transferred Tregs to reverse a disease phenotype does not establish Treg deficiency as a cause of disease.

Another possibility raised in the context of these immunodeficiency states is that a lymphopenic environment may be critical for homeostatic proliferation/activation of dysregulated $\mathrm{T}$ cells. Also, partial immunodeficiency may perturb host defense in such a fashion that the homeostatic burden of gut commensals is abnormal. Stimulation of the innate immune system may interact with abnormal T cells to wreak havoc.

A final hypothesis relates to abnormal homeostasis of peripheral T cells. Impaired TCR signal transduction may alter effector T-cell differentiation and function in multiple ways. It may be that inhibitory feedback loops downstream of TCR triggering are disproportionately impaired in these models such that a weak signal is transmitted but not appropriately downregulated. Alternatively, an appropriate signal to induce anergy is not generated. This group of defects encompasses failure of antigen-specific anergy as well as failure of nonantigen-specific 'self-control'.

Most recently, extensive characterization of the Lat $\mathrm{Y} 136 \mathrm{~F}$ mouse model has produced unexpected results. The transfer of Lat $\mathrm{Y} 136 \mathrm{~F}$ CD4 $\mathrm{T}$ cells into an $\mathrm{MHC} \mathrm{I}^{-/-}$host produces disease [34]. This raises the possibility that participation of Lat in non-TCR signals (in a lymphopenic environment) or ligand-independent tonic TCR signals (in the absence of functional antigen-presenting cells) plays a role. Significantly, proliferating Th2 polarized effector CD4 T cells drive ANA production in wild-type $B$ cells upon adoptive transfer in the absence of a bona fide initiating autoantigen and certainly cannot do so in a cognate manner (in the absence of MHC ॥ molecules).

Most rheumatologists would classify ANA production as 'autoimmune' in nature. It may, however, be important to reassess old assumptions about the antigen-driven etiology of 'autoimmunity' in animal models characterized by ANAs. We have learned in recent years that innate receptors such as the TLRs are required to direct this specificity and now discover that non-specific T-cell help is sufficient to produce ANAs in otherwise normal $B$ cells.

Diseases that develop in the Lat model and in the ZAP-70 allelic series are characterized by $\lg \mathrm{E}$ and Th2 cytokine overproduction. Autoimmunity that sometimes arises in the context of partial human T-cell immunodeficiency is often characterized by IgE production and 'allergic' Th2 diseases. The ZAP-70 allelic series and Lat mouse models bear a much closer resemblance to these relatively rare clinical entities than to common polygenic rheumatic diseases such as RA and SLE. (The reader is referred to an excellent recent review [33].) Nevertheless, this phenomenon raises the possibility that dysregulation of T-cell-intrinsic effector pathways may contribute to disease in some 'classic' rheumatic diseases.

\section{Hyper-responsive T-cell antigen receptor signaling}

We have observed that B-cell hyper-responsiveness appears to be an overwhelming characteristic of murine lupus mouse models. A large number of mice with impaired TCR signaling, with either proximal or distal transduction perturbations, develop dysregulated lymphoid homeostasis and inflammatory diseases [33]. Only a handful of these have been reviewed here, selected because they feature proximal and Tcell-specific perturbations that are more easily interpretable and simplify the cellular mechanisms of disease.

The clearest model to demonstrate that T-cell hyper-responsiveness can also break tolerance may be the $\mathrm{Cbl} / \mathrm{Cbl}-\mathrm{b}$ double-deficient mice. $\mathrm{Cbl}$ and $\mathrm{Cbl}-\mathrm{b}$ are widely expressed E3 ubiquitin ligases that target their substrates for proteosomal degradation [35]. By targeting multiple components of the antigen receptor signal transduction machinery for degradation, $\mathrm{Cbl}$ and $\mathrm{Cbl}-\mathrm{b}$ serve as negative regulators of antigen receptor signaling. Both single and double knockouts (dKOs) have been generated, revealing overlapping as well as developmentally distinct roles in antigen receptor signaling [35]. The T-cell-specific dKO develops a severe systemic disease characterized by arteritis and dsDNA production [36]. $T$ cells are hyper-proliferative and produce large quantities of cytokines in response to TCR stimulation. Yet proximal TCR signaling machinery is differentially affected, with enhanced ZAP-70 phosphorylation but impaired PLC $\gamma 1$ phosphorylation leading to impaired inducible calcium increase. Most interestingly, impaired ligand-induced TCR downmodulation and prolonged Erk phosphorylation characterize dKO T cells. The TCR signaling phenotype is not simply amplified but is qualitatively and kinetically perturbed. Whether the associated disease represents an antigen-specific breach of tolerance or a dysregulated polyclonal response akin to the Lat $\mathrm{Y} 136 \mathrm{~F}$ mice remains unclear.

In contrast to impaired TCR signaling, relatively few mouse models with a 'pure' defect leading to hyper-responsive $T$ cells develop autoimmune disease. One explanation relates to the wiring of TCR signaling machinery and another to the etiology of rheumatic disease. One can a priori engineer hyper-responsive TCR signaling by generating either a hypermorphic allele of a positive regulator or a knockout/ hypomorph of a negative regulator. Although knockouts are easier to generate, negative regulators appear in general to display more functional redundancy than positive regulators of TCR signaling (the opposite may be true in B cells). 
Perhaps these mutants, when generated, have too subtle a phenotype (for example, $\mathrm{Pep}^{-1-}$, to be discussed later) to produce disease on non-autoimmune genetic backgrounds. Another argument relates to the developmental consequences of strong TCR signaling. Examples of dramatically enhanced TCR signaling, including the Lck Y505F mutant and the $\mathrm{Csk}^{-1-}$ mutant, exist [5]. Both have such enhanced TCR signaling that T-cell development in the thymus cannot occur normally due to suppression of RAG expression. In fact, rather than autoimmunity, these types of perturbations cause malignant transformation. In this way, hyper-responsive peripheral $\mathrm{T}$ cells may just be hard to generate with reverse genetics in mice. Alternatively, it may be that only impaired TCR signaling can breach T-cell tolerance without help. Indeed, as we will argue below, hyperresponsive $T$ cells may be a feature of specific autoimmune diseases that require an additional and independent B-cell phenotype. Hyper-responsive T cells, in other words, may not be able to act alone. Perhaps this tells us that our immune system is biased toward protecting us from the ravages of overactive $T$ cells but has fewer built-in defenses against impaired TCR signaling. This might make teleogical sense since the overwhelming evolutionary pressure on the immune system has been infection, not autoimmunity, driving the system to over-reaction, not under-reaction.

\section{Translational data: signaling in $B$ and $T$ cells from patients with rheumatic disease}

Do these mouse models have relevance for human disease? Indeed, perturbations in antigen receptor signal transduction have been identified in lymphocytes from patients with rheumatic diseases.

\section{B cells in human systemic lupus erythematosus}

Stimulation of the BCR on peripheral blood B cells from SLE patients has been reported to cause exaggerated calcium increases, recapitulating functional cellular phenotypes seen in mouse mutants with SLE (for example, Lyn ${ }^{-/-}, \mathrm{Fc \gamma R}_{\mathrm{R}} \mathrm{b}^{-/-}$, and $\mathrm{CD}_{22^{-/-}}$[37]. Significantly, these functional alterations did not correlate with disease activity or with treatment, consistent with a primary pathogenic role. The mechanistic and genetic basis of this phenotype in primary human B cells remains uncertain. Expression of key BCR signaling molecules in SLE $B$ cells has been studied and reduced levels of the negative regulators Lyn and SHIP have been described, reminiscent of SLE mouse models [38]. The convergence of human and mouse data strongly suggests that exaggerated BCR signal transduction, at least in peripheral $B$ cells, may be a fundamental pathogenic feature of human SLE.

\section{T cells in human systemic lupus erythematosus}

Analogous functional studies of $T$ cells from SLE patients have been undertaken. Exaggerated calcium increases in SLE T cells upon TCR stimulation have been reported [39]. Oddly, SLE T cells generally produce reduced quantities of IL-2 [40].
This has been interpreted as an 'anergic' phenotype and might suggest that some of the observed signaling phenomena reflect the influence of a characteristic inflammatory milieu rather than a cell-intrinsic genetic program.

Interestingly, expression levels of TCR $\zeta$ chain were found to be reduced in SLE T cells [39]. The mechanism for this deranged expression is apparently both transcriptional and posttranslational $[40,41]$. Increased expression of the alternative ITAM-bearing receptor FcR $\gamma$ has been observed in those cells, and TCR stimulation results in enhanced FcR phosphorylation. An alternative TCR complex composed of FcR $\gamma$-Syk (replacing $\zeta$-Zap70) has been proposed to account for the altered functional signaling phenotype observed in these cells. Similar $\zeta$ chain downregulation in RA T cells from synovial fluid and in memory $T$ cells has been reported [40].

\section{$\mathbf{T}$ cells in human rheumatoid arthritis}

Interestingly, distinct functional phenotypes have been reported in peripheral blood $T$ cells from patients with RA, including impaired calcium responses and proliferation to TCR stimulation [42]. This is a provocative observation given the T-cell-signaling-impaired Skg mouse, which develops an RA-like clinical phenotype on a susceptible genetic background.

In the end, whether these changes observed in SLE and RA reflect a cell-intrinsic and disease-specific abnormality in $T$ cells or a general change to activated/effector status is less clear, and whether in turn this represents a cause or effect of the inflammatory disease is unknown.

\section{Human genetics}

Functional studies conducted with primary human cells are suggestive but remain correlative. To address cause and effect, human genetics offers some clues. Indeed, numerous human candidate gene association studies have implicated antigen receptor signaling pathways in the pathogenesis of rheumatic diseases. A hypomorphic allele of FcyRllb (lle232Thr) has been associated with SLE in an Asian population [20]. Studies in recent years have also identified disease-associated polymorphisms in CTLA-4, a T-cell inhibitory coreceptor, and mutations that influence splicing and function of CD45 $[43,44]$. Human genetics has seen an explosion in data with the advent of whole genome association studies in the last two years. Unbiased identification of new genetic risk factors for human autoimmune diseases has implicated antigen receptor signaling machinery as well [45]. The B cell SFK Blk and the BCR signaling adaptor BANK1 were identified in recent whole genome scans for lupus $[46,47]$. A single missense polymorphism in PTPN22, a negative regulator of the SFKs, is the second strongest common polymorphism associated with RA outside of the MHC $[48,49]$. Yet the functional consequences of many of these polymorphisms remain unclear. The subtlety of the risk alleles coupled with developmental and network 
complexity of antigen receptor signal transduction make interpretation of phenotypes challenging. How then can polygenic susceptibility loci be studied? How do we move from genetics to pathogenesis?

\section{PTPN22 R620W polymorphism}

An elegant example is the PTPN22 R620W polymorphism, which is associated with multiple autoimmune diseases, including SLE, RA, and type I diabetes [50,51]. The PTPN22 gene product Lyp, the murine ortholog of which is Pep, encodes a hematopoietic cytoplasmic phosphatase. Pep/Lyp negatively regulates TCR signaling by dephosphorylating the activating tyrosine of Lck $[52,53]$. One quarter to one half of Pep is found associated with Csk, a potent negative regulator of TCR signaling which dephosphorylates the inhibitory tyrosine of Lck [54]. Pep cooperatively inhibits TCR signaling by binding Csk and this association in turn is mediated by a proline-rich sequence in the $\mathrm{C}$-terminal region of Pep (PRS1) $[52,54]$.

The R620W polymorphism is located in the critical PRS1 domain of Pep, and impairs the interaction of Pep with Csk $[50,55]$. The risk allele was therefore initially postulated to represent a loss-of-function in which TCR signaling was less effectively inhibited. However, overexpression of the Lyp risk allele in Jurkat cells suggested the opposite (that is, that the risk allele is a gain-of-function, impairing TCR signaling) [55]. A handful of studies of primary human cells from healthy donors as well as patients harboring the risk allele have been published [55-58]. Several appear to confirm the gain-offunction hypothesis but not all are in agreement. In our laboratory, we revisited the question of the functional significance of the R620W risk allele. Functional studies of the wild-type and R619W (murine homolog) Pep alleles in the context of Csk unmasked Pep R619W as a hypomorphic allele (J Zikherman, M Hermiston, D Steiner, K Hasegawa, A Chan, A Weiss,manuscript in preparation).

The $\mathrm{Pep}^{-/-}$mouse confirms Pep as a negative regulator of TCR signaling but no disease phenotype is discernible [59]. Indeed, the Pep null allele appears to require a cooperating mutation to develop disease, just as the R620W polymorphism in humans does not act alone. By crossing the $\mathrm{Pep}^{-/-}$mice onto a background in which hyper-responsive $\mathrm{B}$ cells (characteristic of lupus-prone mice and humans) are active, we have been able to generate a mouse model in which a bona fide human genetic risk factor produces a lupus-like disease. In $\mathrm{Pep}^{-/-}$CD45 E613R double-mutant animals, hyper-responsive $\mathrm{Pep}^{-/-} \mathrm{T}$ cells and hyper-responsive CD45 E613R B cells cooperate to break tolerance (J Zikherman, M Hermiston, D Steiner, K Hasegawa, A Chan, A Weiss, manuscript in preparation). Definitive functional conclusions about the R620W allele will depend on future studies of a knock-in mouse. Even those will then have to be pursued in the context of cooperating mutations in order to recapitulate human disease pathogenesis.

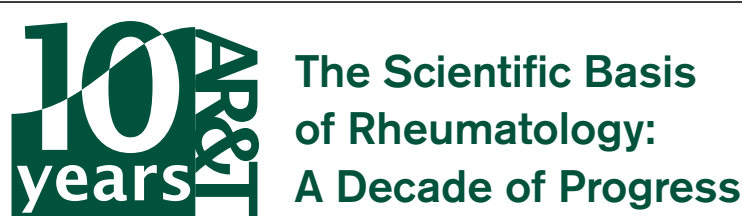

This article is part of a special collection of reviews, The Scientific Basis of Rheumatology: A Decade of Progress, published to mark Arthritis Research \& Therapy's 10th anniversary.

Other articles in this series can be found at: http://arthritis-research.com/sbr

\section{Conclusion}

Conventional antigen-driven autoimmune disease with pathogenic clones is most clearly observed in the organ-specific autoimmune endocrinopathies, including insulin-dependent diabetes mellitus, autoimmune ovarian failure, and others. Certainly, polyendocrinopathy syndromes, and possibly sporadic variants of these diseases, appear to be characterized by failure of central tolerance [60]. However, more and more questions have been raised regarding the 'autoimmune' nature of diseases arising in the setting of partial immunodeficiency. The common systemic rheumatic diseases, particularly SLE and RA, are under scrutiny as well. Conventional models of disease pathogenesis are being revised as the complex interplay of innate and adaptive pathways is being dissected and appreciated. Are alterations in antigen receptor signaling pathways significant in disease pathogenesis? Overwhelming data suggest that they are. Are specific auto-antigens required to break tolerance and drive disease? This is less clear. We have tried in this review to demonstrate the power of combining genetic and functional studies in mice and humans and to point out the limits of our current understanding. Much remains to be understood in the pathogenesis of rheumatic diseases, and new targets for therapy will no doubt emerge.

\section{Competing interests}

The authors declare that they have no competing interests.

\section{Acknowledgments}

This work was supported in part by a post-doctoral grant from the Arthritis Foundation (to JZ).

\section{References}

1. Abraham RT, Weiss A: Jurkat T cells and development of the Tcell receptor signalling paradigm. Nat Rev Immunol 2004, 4: 301-308.

2. Tomlinson MG, Lin J, Weiss A: Lymphocytes with a complex: adapter proteins in antigen receptor signaling. Immunol Today 2000, 21:584-591.

3. Koretzky GA, Abtahian F, Silverman MA: SLP76 and SLP65: complex regulation of signalling in lymphocytes and beyond. Nat Rev Immunol 2006, 6:67-78.

4. Acuto $\mathrm{O}$, Bartolo VD, Michel F: Tailoring T-cell receptor signals 
by proximal negative feedback mechanisms. Nat Rev Immunol 2008, 8:699-712.

5. Hermiston ML, Xu Z, Majeti R, Weiss A: Reciprocal regulation of lymphocyte activation by tyrosine kinases and phosphatases. $J$ Clin Invest 2002, 109:9-14.

6. Niiro H, Clark EA: Regulation of B-cell fate by antigen-receptor signals. Nat Rev Immunol 2002, 2:945-956.

7. Billadeau DD, Leibson PJ: ITAMs versus ITIMs: striking a balance during cell regulation. J Clin Invest 2002, 109:161168.

8. Buhl AM, Cambier JC: Co-receptor and accessory regulation of B-cell antigen receptor signal transduction. Immunol Rev 1997, 160:127-138.

9. Carreno BM, Collins M: The B7 family of ligands and its receptors: new pathways for costimulation and inhibition of immune responses. Annu Rev Immunol 2002, 20:29-53.

10. $\mathrm{Xu} Y$, Harder KW, Huntington ND, Hibbs ML, Tarlinton DM: Lyn tyrosine kinase: accentuating the positive and the negative. Immunity 2005, 22:9-18.

11. Malissen $B$, Malissen M: Functions of TCR and pre-TCR subunits: lessons from gene ablation. Curr Opin Immunol 1996, 8: 383-393.

12. Benschop RJ, Cambier JC: B cell development: signal transduction by antigen receptors and their surrogates. Curr Opin Immunol 1999, 11:143-151.

13. Fontenot JD, Rudensky AY: A well adapted regulatory contrivance: regulatory $\mathrm{T}$ cell development and the forkhead family transcription factor Foxp3. Nat Immunol 2005, 6:331337.

14. Zouali M, Sarmay G: B lymphocyte signaling pathways in systemic autoimmunity: implications for pathogenesis and treatment. Arthritis Rheum 2004, 50:2730-2741.

15. Sato S, Ono N, Steeber DA, Pisetsky DS, Tedder TF: CD19 regulates B lymphocyte signaling thresholds critical for the development of B-1 lineage cells and autoimmunity. J Immunol 1996, 157:4371-4378.

16. Majeti R, Xu Z, Parslow TG, Olson JL, Daikh DI, Killeen N, Weiss $A$ : An inactivating point mutation in the inhibitory wedge of CD45 causes lymphoproliferation and autoimmunity. Cell 2000, 103:1059-1070.

17. Hermiston ML, Tan AL, Gupta VA, Majeti R, Weiss A: The juxtamembrane wedge negatively regulates CD45 function in B cells. Immunity 2005, 23:635-647.

18. O'Keefe TL, Williams GT, Batista FD, Neuberger MS: Deficiency in CD22, a B cell-specific inhibitory receptor, is sufficient to predispose to development of high affinity autoantibodies. J Exp Med 1999, 189:1307-1313.

19. Christensen SR, Shlomchik MJ: Regulation of lupus-related autoantibody production and clinical disease by Toll-like receptors. Semin Immuno/ 2007, 19:11-23.

20. Tarasenko T, Dean JA, Bolland S: FcgammaRIIB as a modulator of autoimmune disease susceptibility. Autoimmunity 2007, 40: 409-417.

21. Yu CC, Tsui HW, Ngan BY, Shulman MJ, Wu GE, Tsui FW: B and $T$ cells are not required for the viable motheaten phenotype. J Exp Med 1996, 183:371-380.

22. Fukuyama H, Nimmerjahn F, Ravetch JV: The inhibitory Fcgamma receptor modulates autoimmunity by limiting the accumulation of immunoglobulin $\mathrm{G}^{+}$anti-DNA plasma cells. Nat Immunol 2005, 6:99-106.

23. Wardemann H, Yurasov S, Schaefer A, Young JW, Meffre E, Nussenzweig MC: Predominant autoantibody production by early human B cell precursors. Science 2003, 301:1374-1377.

24. Mohan C, Morel L, Yang P, Wakeland EK: Genetic dissection of systemic lupus erythematosus pathogenesis: Sle2 on murine chromosome 4 leads to B cell hyperactivity. J Immunol 1997, 159:454-465.

25. Mohan C, Alas E, Morel L, Yang P, Wakeland EK: Genetic dissection of SLE pathogenesis. Sle1 on murine chromosome 1 leads to a selective loss of tolerance to H2A/H2B/DNA subnucleosomes. J Clin Invest 1998, 101:1362-1372.

26. Kumar KR, Li L, Yan M, Bhaskarabhatla M, Mobley AB, Nguyen C, Mooney JM, Schatzle JD, Wakeland EK, Mohan C: Regulation of B cell tolerance by the lupus susceptibility gene Ly108. Science 2006, 312:1665-1669.

27. Sakaguchi N, Takahashi T, Hata H, Nomura T, Tagami T, Yamazaki S, Sakihama T, Matsutani T, Negishi I, Nakatsuru S, Sakaguchi S:
Altered thymic T-cell selection due to a mutation of the ZAP70 gene causes autoimmune arthritis in mice. Nature 2003, 426:454-460.

28. Sakaguchi S, Sakaguchi N, Yoshitomi H, Hata H, Takahashi T, Nomura T: Spontaneous development of autoimmune arthritis due to genetic anomaly of T cell signal transduction: Part 1. Semin Immunol 2006, 18:199-206.

29. Yoshitomi H, Sakaguchi N, Kobayashi K, Brown GD, Tagami T, Sakihama T, Hirota K, Tanaka S, Nomura T, Miki I, Gordon S, Akira $S$, Nakamura T, Sakaguchi S: A role for fungal \{beta\}-glucans and their receptor Dectin-1 in the induction of autoimmune arthritis in genetically susceptible mice. J Exp Med 2005, 201: 949-960.

30. Siggs OM, Miosge LA, Yates AL, Kucharska EM, Sheahan D, Brdicka T, Weiss A, Liston A, Goodnow CC: Opposing functions of the T cell receptor kinase ZAP-70 in immunity and tolerance differentially titrate in response to nucleotide substitutions. Immunity 2007, 27:912-926.

31. Sommers CL, Park CS, Lee J, Feng C, Fuller CL, Grinberg A, Hildebrand JA, Lacaná E, Menon RK, Shores EW, Samelson LE, Love PE: A LAT mutation that inhibits T cell development yet induces lymphoproliferation. Science 2002, 296:2040-2043.

32. Aguado $E$, Richelme $S$, Nuñez-Cruz $S$, Miazek A, Mura AM, Richelme M, Guo XJ, Sainty D, He HT, Malissen B, Malissen M: Induction of $\mathrm{T}$ helper type 2 immunity by a point mutation in the LAT adaptor. Science 2002, 296:2036-2040.

33. Liston A, Enders A, Siggs OM: Unravelling the association of partial T-cell immunodeficiency and immune dysregulation. Nat Rev Immunol 2008, 8:545-558.

34. Wang $Y$, Kissenpfennig A, Mingueneau M, Richelme S, Perrin $P$, Chevrier S, Genton C, Lucas B, DiSanto JP, Acha-Orbea H, Malissen B, Malissen M: Th2 lymphoproliferative disorder of LatY136F mutant mice unfolds independently of TCR-MHC engagement and is insensitive to the action of Foxp $3^{+}$regulatory T cells. J Immuno/ 2008, 180:1565-1575.

35. Duan L, Reddi AL, Ghosh A, Dimri M, Band H: The Cbl family and other ubiquitin ligases: destructive forces in control of antigen receptor signaling. Immunity 2004, 21:7-17.

36. Naramura M, Jang IK, Kole H, Huang F, Haines D, Gu H: c-Cbl and $\mathrm{Cbl}-\mathrm{b}$ regulate $\mathrm{T}$ cell responsiveness by promoting ligand-induced TCR down-modulation. Nat Immunol 2002, 3: 1192-1199.

37. Liossis SN, Kovacs B, Dennis G, Kammer GM, Tsokos GC: B cells from patients with systemic lupus erythematosus display abnormal antigen receptor-mediated early signal transduction events. J Clin Invest 1996, 98:2549-2557.

38. Pugh-Bernard AE, Cambier JC: $B$ cell receptor signaling in human systemic lupus erythematosus. Curr Opin Rheumatol 2006, 18:451-455.

39. Liossis SN, Ding XZ, Dennis GJ, Tsokos GC: Altered pattern of TCR/CD3-mediated protein-tyrosyl phosphorylation in T cells from patients with systemic lupus erythematosus. Deficient expression of the $\mathrm{T}$ cell receptor zeta chain. J Clin Invest 1998, 101:1448-1457.

40. Ciszak L, Pawlak E, Kosmaczewska A, Potoczek S, Frydecka I: Alterations in the expression of signal-transducing CD3 zeta chain in $T$ cells from patients with chronic inflammatory/autoimmune diseases. Arch Immunol Ther Exp (Warsz) 2007, 55:373-386.

41. Tenbrock K, Juang YT, Kyttaris VC, Tsokos GC: Altered signal transduction in SLE T cells. Rheumatology (Oxford) 2007, 46: 1525-1530.

42. Allen ME, Young SP, Michell RH, Bacon PA: Altered T lymphocyte signaling in rheumatoid arthritis. Eur J Immunol 1995, 25: 1547-1554.

43. Gough SC, Walker LS, Sansom DM: CTLA4 gene polymorphism and autoimmunity. Immunol Rev 2005, 204:102-115.

44. Tchilian EZ, Beverley PC: Altered CD45 expression and disease. Trends Immunol 2006, 27:146-153.

45. Xavier RJ, Rioux JD: Genome-wide association studies: a new window into immune-mediated diseases. Nat Rev Immunol 2008, 8:631-643.

46. Kozyrev SV, Abelson AK, Wojcik J, Zaghlool A, Linga Reddy MV, Sanchez E, Gunnarsson I, Svenungsson E, Sturfelt G, Jönsen A, Truedsson L, Pons-Estel BA, Witte T, D'Alfonso S, Barizzone N, Danieli MG, Gutierrez C, Suarez A, Junker P, Laustrup H, González-Escribano MF, Martin J, Abderrahim H, Alarcón- 
Riquelme ME: Functional variants in the B-cell gene BANK1 are associated with systemic lupus erythematosus. Nat Genet 2008, 40:211-216.

47. Hom G, Graham RR, Modrek B, Taylor KE, Ortmann W, Garnier S, Lee AT, Chung SA, Ferreira RC, Pant PV, Ballinger DG, Kosoy R, Demirci FY, Kamboh Ml, Kao AH, Tian C, Gunnarsson I, Bengtsson AA, Rantapää-Dahlqvist S, Petri M, Manzi S, Seldin MF, Rönnblom L, Syvänen AC, Criswell LA, Gregersen PK, Behrens TW: Association of Systemic Lupus Erythematosus with C8orf13-BLK and ITGAM-ITGAX. N Engl J Med 2008, 358: 900-909.

48. Plenge RM, Seielstad M, Padyukov L, Lee AT, Remmers EF, Ding B, Liew A, Khalili H, Chandrasekaran A, Davies LR, Li W, Tan AK, Bonnard C, Ong RT, Thalamuthu A, Pettersson S, Liu C, Tian C Chen WV, Carulli JP, Beckman EM, Altshuler D, Alfredsson L, Criswell LA, Amos Cl, Seldin MF, Kastner DL, Klareskog L, Gregersen PK: TRAF1-C5 as a risk locus for rheumatoid arthritisa genomewide study. N Engl J Med 2007, 357:1199-1209.

49. Wellcome Trust Case Control Consortium: Genome-wide association study of 14,000 cases of seven common diseases and 3,000 shared controls. Nature 2007, 447:661-678.

50. Begovich AB, Carlton VE, Honigberg LA, Schrodi SJ, Chokkalingam AP, Alexander HC, Ardlie KG, Huang Q, Smith AM, Spoerke JM, Conn MT, Chang M, Chang SY, Saiki RK, Catanese JJ, Leong DU, Garcia VE, McAllister LB, Jeffery DA, Lee AT, Batliwalla F, Remmers E, Criswell LA, Seldin MF, Kastner DL, Amos $\mathrm{Cl}$, Sninsky JJ, Gregersen PK: A missense single-nucleotide polymorphism in a gene encoding a protein tyrosine phosphatase (PTPN22) is associated with rheumatoid arthritis. Am $J$ Hum Genet 2004, 75:330-337.

51. Gregersen PK, Lee HS, Batliwalla F, Begovich AB: PTPN22: setting thresholds for autoimmunity. Semin Immunol 2006, 18: 214-223.

52. Cloutier JF, Veillette A: Cooperative inhibition of T-cell antigen receptor signaling by a complex between a kinase and a phosphatase. J Exp Med 1999, 189:111-121.

53. Gjorloff-Wingren A, Saxena M, Williams S, Hammi D, Mustelin T: Characterization of TCR-induced receptor-proximal signaling events negatively regulated by the protein tyrosine phosphatase PEP. Eur J Immunol 1999, 29:3845-3854.

54. Cloutier JF, Veillette A: Association of inhibitory tyrosine protein kinase p50csk with protein tyrosine phosphatase PEP in T cells and other hemopoietic cells. EMBO J 1996, 15: 4909-4918.

55. Vang $T$, Congia M, Macis MD, Musumeci L, Orrú V, Zavattari $P$, Nika K, Tautz L, Taskén K, Cucca F, Mustelin T, Bottini N: Autoimmune-associated lymphoid tyrosine phosphatase is a gain-offunction variant. Nat Genet 2005, 37:1317-1319.

56. Rieck M, Arechiga A, Onengut-Gumuscu S, Greenbaum C, Concannon $\mathrm{P}$, Buckner JH: Genetic variation in PTPN22 corresponds to altered function of $\mathrm{T}$ and $\mathrm{B}$ lymphocytes. J Immunol 2007, 179:4704-4710.

57. Aarnisalo J, Treszl A, Svec $P$, Marttila J, Oling V, Simell O, Knip M, Körner A, Madacsy L, Vasarhelyi B, llonen J, Hermann R: Reduced $\mathrm{CD}^{+}{ }^{+} \mathrm{T}$ cell activation in children with type 1 diabetes carrying the PTPN22/Lyp 620Trp variant. J Autoimmun 2008, 31:13-21.

58. Lefvert AK, Zhao $Y$, Ramanujam R, Yu S, Pirskanen R, Hammarstrom L: PTPN22 R620W promotes production of antiAChR autoantibodies and IL-2 in myasthenia gravis. J Neuroimmunol 2008, 197:110-113.

59. Hasegawa K, Martin F, Huang G, Tumas D, Diehl L, Chan AC: PEST domain-enriched tyrosine phosphatase (PEP) regulation of effector/memory T cells. Science 2004, 303:685-689.

60. DeVoss JJ, Anderson MS: Lessons on immune tolerance from the monogenic disease APS1. Curr Opin Genet Dev 2007, 17: 193-200. 